\title{
TYPE SPECIMENS OF SCHLECHTER'S NAMES IN ORCHIDACEAE AT THE CONSERVATOIRE ET JARDIN BO'TANIQUES, GENEVE.
}

\author{
D. F. BLAXell
}

(Accepted 15.7.1977)

\begin{abstract}
Blaxell, D. F. (National Herbarium of New South Wales, Royal Botanic Gardens, Sydney, Australia 2000) 1978. Type specimens of Schlechter's names in Orchidaceae at the Conservatoire et Jardin Botaniques, Genève. Telopea 1 (5): 359-363-In the Geneva Herbarium most specimens of Orchidaceae collected by Rudolf Schlechter in Asia and Africa between 1898 and 1910 are Types.
\end{abstract}

\section{INTRODUCTION}

In previous papers (Blaxell 1973, 1975) I listed some 266 Isotypes and Isosyntypes of Schlechter's published names of orchids from various countries. Whilst holding the position of Australian Botanical Liaison Officer at the Royal Botanic Gardens, Kew, in 1974-75 I examined collections by Schlechter in other herbaria and checked them for Type status. The following list is for Geneva (G) and comprises names in the larger genera only, since shortage of time necessitated a selection of genera for examination.

Schlechter apparently distributed many duplicates of each of his numbers of Orchidaceae, resulting in quite rich accumulations of Isotypes and Isosyntypes in many of the world's herbaria. Since the Holotypes were destroyed in Berlin in 1943, these duplicate specimens are of importance in current taxonomic studies of Asian and African orchids. I have not attempted to lectotypify any of Schlechter's names as that requires more detailed study of all the duplicates. I am only listing specimens and indicating their location and status.

The Table format follows that of the previous papers, except for the omission of places of publication in this listing. These can be obtained readily from Index Kewensis.

It should be noted that the majority of specimens listed here are ex "Herb. Delessert." Also, the collector's number on some of the original labels has been written over in an apparent attempt to render it more legible, but this has resulted in a greater degree of illegibility than the original!

I wish to thank the Director of the Conservatoire et Jardin Botaniques, Professor Jacques Miège, for allowing me to examine the extensive collections held there, and also Miss Adelaide Stork for her kind assistance in the Herbarium. 
Vol. 1 (5): 1978

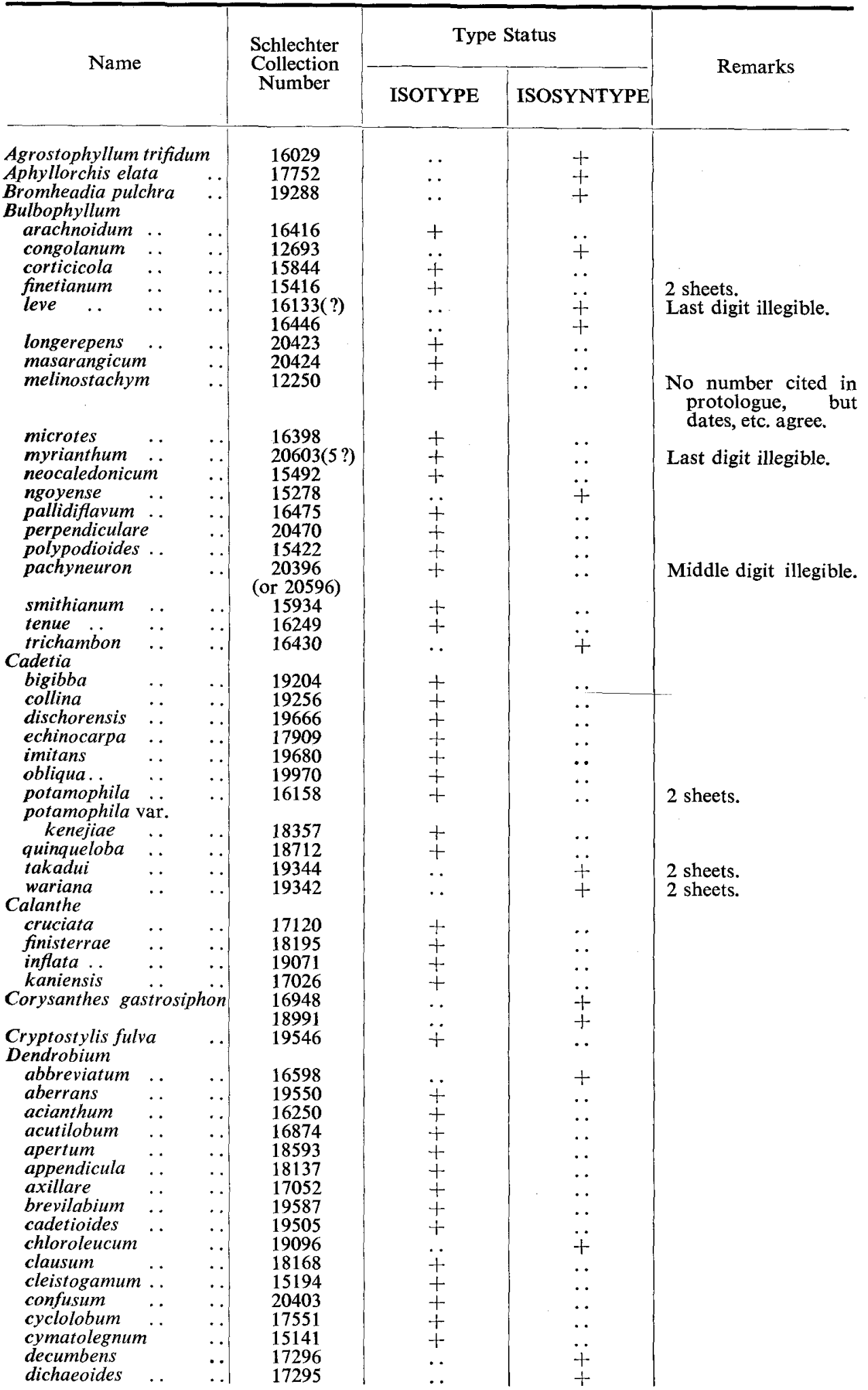




\begin{tabular}{|c|c|c|c|c|c|}
\hline \multirow{2}{*}{\multicolumn{2}{|c|}{ Name }} & \multirow{2}{*}{$\begin{array}{l}\text { Schlechter } \\
\text { Collection } \\
\text { Number }\end{array}$} & \multicolumn{2}{|c|}{ Type Status } & \multirow{2}{*}{ Remarks } \\
\hline & & & ISOTYPE & ISOSYNTYPE & \\
\hline \multicolumn{6}{|l|}{ Dendrobium } \\
\hline $\begin{array}{l}\text { discocaulon } \\
\text { disoides }\end{array}$ & .. & 19320 & . & + & \\
\hline $\begin{array}{l}\text { disoides } \\
\text { diamuense }\end{array}$ & .. & 17118 & + & $\cdots$ & \\
\hline djamuense & .. & 16822 & + & .. & \\
\hline $\begin{array}{l}\text { dryadum } \\
\text { eitapense }\end{array}$ & . & 18211 & + & $\cdots$ & \\
\hline $\begin{array}{l}\text { eitapense } \\
\text { eleutherogloss }\end{array}$ & $\ddot{u m}$ & $\begin{array}{l}12976 \\
15593\end{array}$ & + & $\ddot{t}$ & \\
\hline extra-axillare & & 16491 & $\ddot{+}$ & 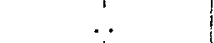 & \\
\hline fariniferum & .. & $\begin{array}{l}16526 \\
17817\end{array}$ & $\because$ & + & \\
\hline finetianum & .. & 15505 & $\ddot{+}$ & $\begin{array}{l}+ \\
.\end{array}$ & 2 sheets. \\
\hline fagellum & $\ldots$ & 18402 & + & $\cdot \cdot$ & \\
\hline fornicatum & $\therefore$ & 19539 & + & $\therefore$ & \\
\hline gatiense & .. & 16991 & + & $\cdots$ & \\
\hline globiflorum & .. & 17141 & + & $\because$ & 3 sheets. \\
\hline grossum & . & 19073 & .. & + & \\
\hline hamadryas & & 17336 & + & .. & \\
\hline hippocrepiferu & & 18736 & + & $\cdots$ & \\
\hline & & $\begin{array}{l}20000 \\
17799\end{array}$ & $t$ & $\ddot{+}$ & \\
\hline $\begin{array}{l}\text { iboense } \\
\text { isomerum }\end{array}$ & $\begin{array}{l}\cdots \\
. .\end{array}$ & 15980 & $\ddot{+}$ & $\ldots$ & \\
\hline jadunae & .. & 19332 & + & .. & \\
\hline lamproglossum & & 18544 & + & .. & \\
\hline leopardinum & & 18023 & & $\cdots$ & $\begin{array}{l}\text { Later homonym; non } \\
\text { Wallich } 1824 \text {. }\end{array}$ \\
\hline $\begin{array}{l}\text { leucohybos var } \\
\text { leucanthum }\end{array}$ & & 17486 & .. & + & \\
\hline $\begin{array}{l}\text { litorale. } \\
\text { lockhartioides }\end{array}$ & & 19958 & $\because$ & + & \\
\hline lockhartioides & & 20447 & + & $\therefore$ & $\begin{array}{l}\text { No number cited in } \\
\text { protologue, other } \\
\text { details agree. }\end{array}$ \\
\hline loesenerianum & & 20280 & + & $\cdots$ & \\
\hline lucidum & & 19712 & + & .. & 2 sheets. \\
\hline meliodorum & . & 17888 & + & . & \\
\hline minjemense & $\cdots$ & 16245 & + & $\ddot{r}$ & \\
\hline mischobulbum & & $\begin{array}{l}18030 \\
18958\end{array}$ & .. & + & \\
\hline $\begin{array}{l}\text { musciferum } \\
\text { nardoides }\end{array}$ & . & $\begin{array}{l}18958 \\
18722(9 ?)\end{array}$ & $\ddot{+} ?$ & . & Last digit illegible. \\
\hline $\begin{array}{l}\text { nardoides } \\
\text { obliquum }\end{array}$ & $\because$ & $\begin{array}{l}18722(9 ?) \\
18882\end{array}$ & + & $\because$ & Last aigit iliegioie. \\
\hline obovatum & .. & 16656 & + & .. & \\
\hline odontopus & .. & 17248 & + & .. & \\
\hline odoratum & . & 20457 & + & . & \\
\hline oreodoxa & . & 18804 & + & .. & \\
\hline pachystele & . & 19182 & + & .. & \\
\hline patulum & .. & 17987 & + & .. & \\
\hline pemae ... & .. & 19908 & + & .. & \\
\hline perlongum & . & 17102 & + & .. & \\
\hline petiolatum & . & 18710 & + & .. & \\
\hline $\begin{array}{l}\text { piestocaulon } \mathrm{v} \\
\text { kauloense }\end{array}$ & var. & 16831 & + & & \\
\hline $\begin{array}{l}\text { kauloense } \\
\text { pleianthum }\end{array}$ & $\because$ & 16303 & + & $\because$ & 2 sheets. \\
\hline pleurodes & $\therefore$ & 19580 & + & .. & \\
\hline pluricostatum & & 19269 & + & .. & \\
\hline poissonianum & & 15590 & + & .. & \\
\hline polyschistum & & 20279 & + & 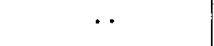 & \\
\hline $\begin{array}{l}\text { poneroides var } \\
\text { angustum }\end{array}$ & & 16688 & + & & \\
\hline potamophila & & 16482 & + & .. & 2 sheets. \\
\hline prostheciglosst & & 18174 & + & 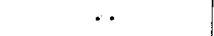 & \\
\hline $\begin{array}{l}\text { prostheciglossu } \\
\text { obtusilobum }\end{array}$ & sum var. & 18805 & + & & \\
\hline pumilio & & 17998 & .. & $\ddot{+}$ & 2 sheets. \\
\hline & & 16545 & .. & + & \\
\hline quadriferum & . & 17143 & + & & \\
\hline $\begin{array}{l}\text { regale } \\
\text { rhytidothece }\end{array}$ & $\because$ & $\begin{array}{l}19068 \\
19518\end{array}$ & $\ddot{t}$ & + & \\
\hline
\end{tabular}


Vol. 1 (5): 1978

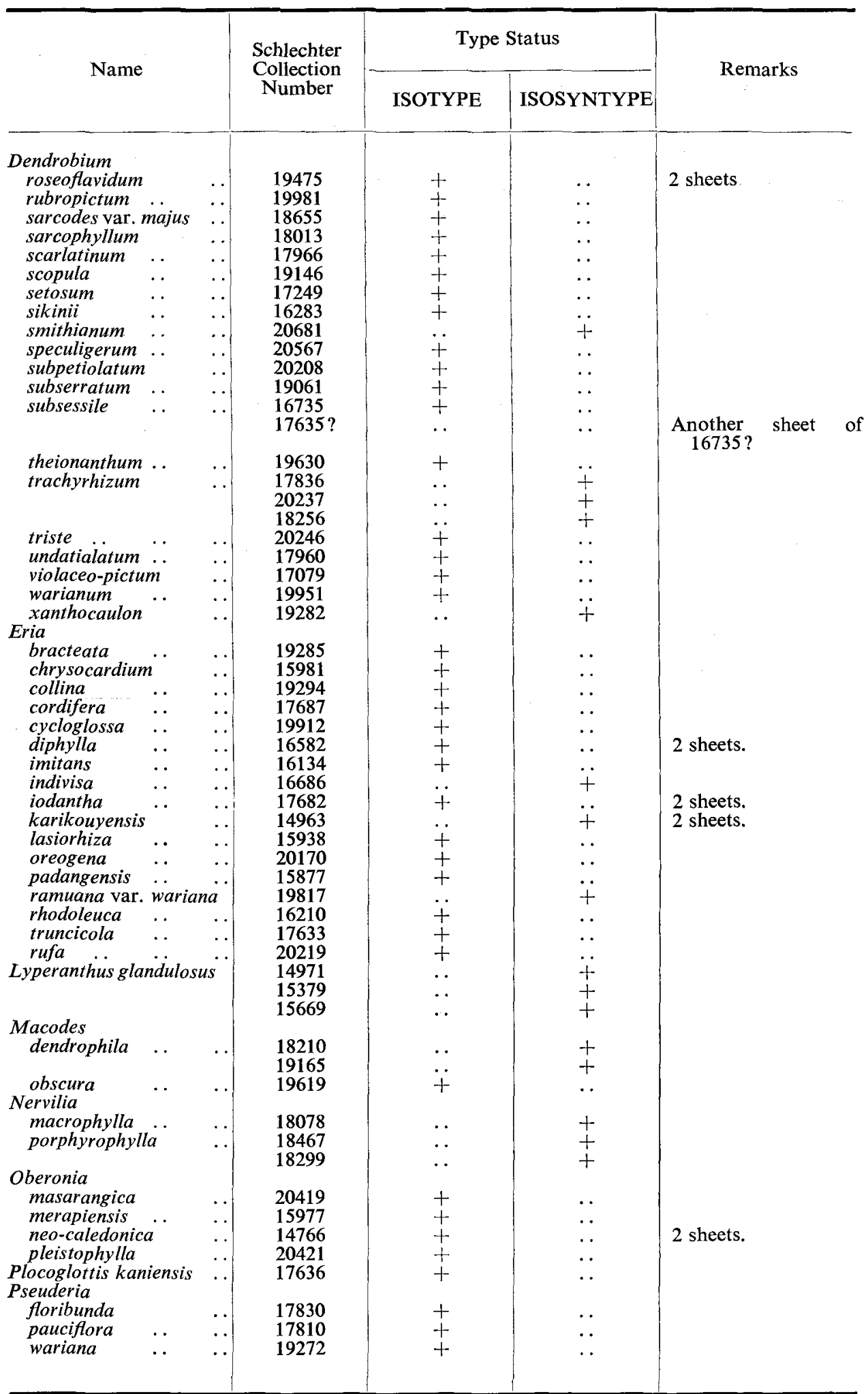




\section{LITERATURE CITED}

Blaxell, D. F., 1973-The Status of Schlechter's specimens of Orchidaceae held at the National Herbarium of New South Wales-1. New Guinea. Contr. New South Wales Natl. Herb. 4 (7): 457-469.

Blaxell, D. F., 1975-The Status of Schlechter's specimens of Orchidaceae held at the National Herbarium of New South Wales-2. New Caledonia, Celebes, Borneo, Sumatra. Telopea 1 (1): 49-54. 